\title{
1 ASM-Clust: classifying functionally diverse protein families using alignment score matrices
}

2 Daan R. Speth ${ }^{1}$ and Victoria J. Orphan ${ }^{1}$

3

$4{ }^{1}$ Division of Geological and Planetary Sciences, California Institute of Technology, Pasadena, 5 CA, USA

7 Correspondence:

8 dspeth@caltech.edu or vorphan@gps.caltech.edu

\section{Abstract}

11 Rapid advances in sequencing technology have resulted in the availability of genomes from 12 organisms across the tree of life. Accurately interpreting the function of proteins in these 13 genomes is a major challenge, as annotation transfer based on homology frequently results in

14 misannotation and error propagation. This challenge is especially pressing for organisms whose 15 genomes are directly obtained from environmental samples, as interpretation of their physiology 16 and ecology is often based solely on the genome sequence. For complex protein (super)families 17 containing a large number of sequences, classification can be used to determine whether 18 annotation transfer is appropriate, or whether experimental evidence for function is lacking. Here 19 we present a novel computational approach for de novo classification of large protein 20 (super)families, based on clustering an alignment score matrix obtained by aligning all sequences 21 in the family to a small subset of the data. We evaluate our approach on the enolase family in the 22 Structure Function Linkage Database.

24 Availability and implementation

25 ASM-Clust is implemented in bash with helper scripts in perl. Scripts comprising ASM-Clust are 26 available for download from https://github.com/dspeth/bioinfo_scripts/tree/master/ASM_clust/ 


\section{Introduction}

29 The rapid advances in sequencing technology have led to a dramatic increase in available genome sequences. This genomic data has provided new perspectives on big questions in biology, such as the diversity of life, the distribution of metabolic traits across the tree of life, and the origin of eukaryotes (Hug et al. 2016; Zaremba-Niedzwiedzka et al. 2017; Borrel et al. 2019). In addition, each newly available genome sequence contains novel protein sequences, yielding novel protein families of unknown function and expanding families with previously characterized representatives. Automatic functional annotation of novel protein sequences is generally done by annotation transfer from known homologous proteins, either using sequence alignment or hidden markov models (Altschul et al. 1990; Finn, Clements, and Eddy 2011). This approach can, and often does, result in misinterpretation of the function of proteins in mechanistically diverse superfamilies, and is prone to subsequent error propagation (Schnoes et al. 2009). Accurately interpreting the function of novel proteins is one of the grand challenges in biology, and relies heavily on availability of experimental data. Classifying mechanistically diverse protein superfamilies provides insight in knowledge gaps, can indicate whether annotation transfer is appropriate, and can help guide future experiments.

44 There are various automatic tools available for classification of proteins into isofunctional 45 families using sequence similarity, active site characteristics, and phylogenetic relationships 46 (Brown, Krishnamurthy, and Sjölander 2007; Lee, Rentzsch, and Orengo 2010; de Melo-

47 Minardi, Bastard, and Artiguenave 2010; Leuthaeuser et al. 2016; Knutson et al. 2017). 48 Alternatively, the structure of a protein family can be interactively explored using sequence 49 similarity networks (SSNs) (Atkinson et al. 2009; Copp et al. 2018). SSNs are constructed based on pairwise all vs all alignment, with each node in the network representing a sequence, and each edge between two nodes representing the alignment between sequences. Clusters of nodes can be manually selected, or identified using a clustering algorithm such as MCL (Enright, Van

53 Dongen, and Ouzounis 2002). SSNs are a powerful method to investigate protein families, but

54 the network visualization limits the number of sequences that can be included, and alignments 55 between separate domains of multi-domain proteins may confuse the analysis.

56 Here we present ASM-Clust, an alternative method that uses alignment score matrix (ASM)

57 clustering. For each input sequence, ASM-Clust generates a profile consisting of a large number 
59 each sequence. For a dataset containing $N$ sequences, alignments are generated for all $\mathrm{N}$

60 sequences against a randomly selected subset of $n$ sequences, and taking each alignment score, or

61 a zero if the sequence did not align to the reference. This results in a matrix of $N \times n$ values

62 which is subsequently visualized using t-distributed stochastic neighbor embedding (t-SNE)

63 (Van der Maaten and Hinton 2008; Van der Maaten 2014), and can be clustered using DBscan

64 (Ester et al. 1996).

\section{Implementation}

67 ASM-Clust is implemented in bash with helper scripts in perl, and will take a protein fasta file as

68 the sole input. Fasta files are processed with ASM_clust.sh, which 1) randomly selects a subset

69 of $\mathrm{n}$ sequences (default 1000), 2) aligns the entire dataset to the subset of $\mathrm{n}$ sequences, 3)

70 combines all scores into a matrix (inserting 0 for query-database pairs that did not produce an

71 alignment), and 4) reduces the matrix to 2 dimensions using t-SNE (Figure 1a). For flexible

72 usage, ASM-Clust supports alignment using DIAMOND (Buchfink, Xie, and Huson 2015),

73 BLAST (Altschul et al. 1990), or MMSeqs2 (Steinegger and Söding 2017), and uses MMSeqs2

74 as default alignment software. Clustering results are comparable between different alignment

75 software (Supplemental Figure S1). Other user-defined options are the number of sequences in

76 the subset (default 1000), the main t-SNE parameter "perplexity" (default 1000) and maximum

77 iterations (default 5000) for dimensionality reduction, and the number of threads used by the

78 alignment software (default 1). Although the clustering is generally similar with multiple

79 randomly chosen subsets (Supplemental Figure S2), the subset can be defined for reproducibility.

80 The output of ASM_clust.sh can be visualized as a scatterplot where each dot represents a

81 sequence, and clusters are readily apparent (Figure 1b, Supplemental figure S1-S3). This format

82 allows additional annotation with sequence features, such as taxonomy, length, or composition.

83 The visualization in Figure 1b and Supplementary Figures S1-S3 was created using R, with the

84 ggplot2 package, and clusters were called using the dbscan package. The t-SNE result and the 85 annotation data downloaded from SFLD were combined prior to visualization. Clusters obtained 86 with ASM-Clust can be further refined by iteratively applying the method to a subset of poorly 87 resolved data, such as the "hub"s cluster (Supplementary Figure S3). The smaller total number of 88 sequences in the second iteration, combined with lowering the perplexity value of the t-SNE, 
89 increases the resolving power of the analysis for clades with a small number of sequences, thus

90 resolving rare classes with few members (Supplementary Figure S3).

91

92

A 1) Subset $n$ sequences

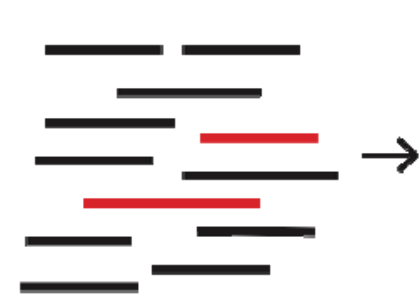

2) Align $N$ sequences to subset $n$
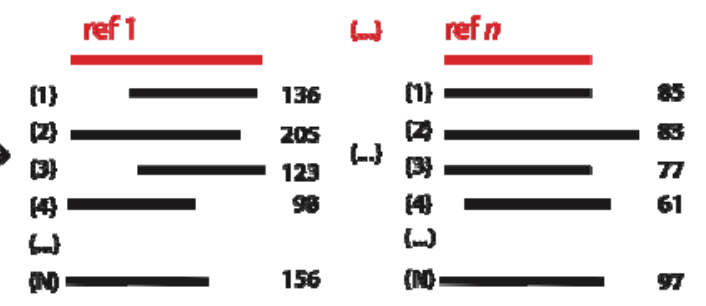

\section{3] Bulld $N \times n$ Matrix}

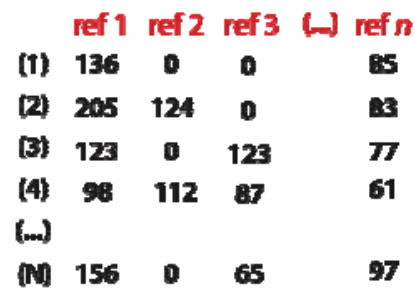

B
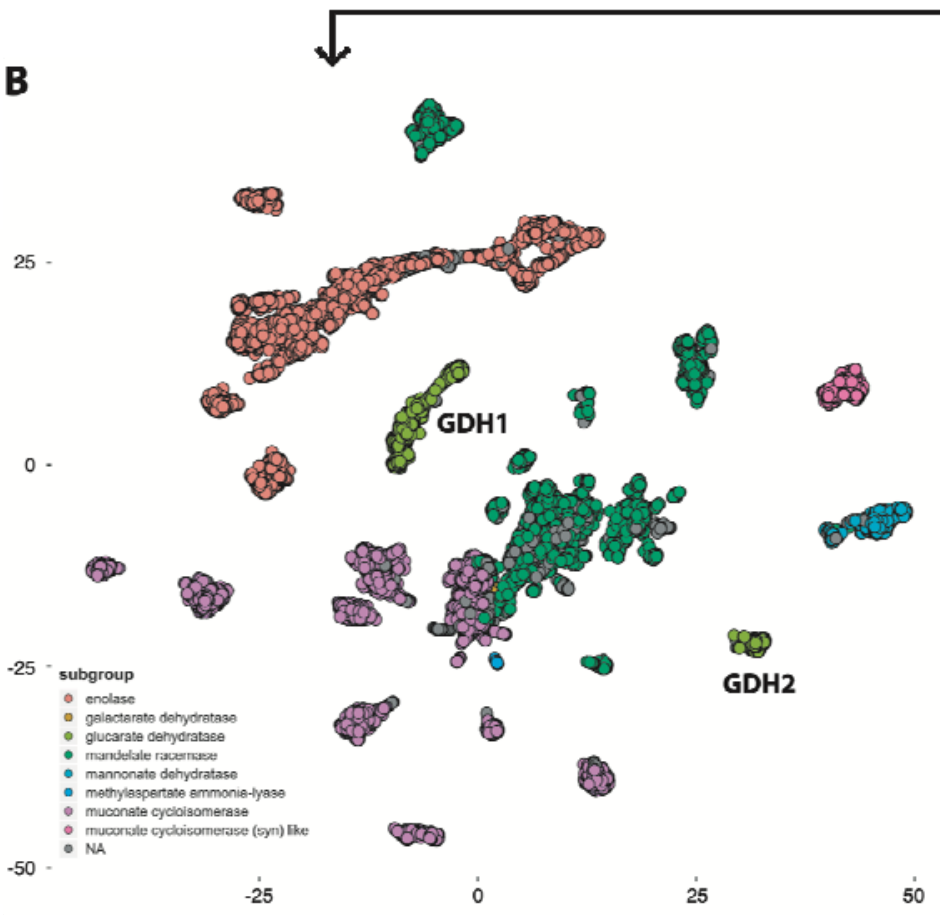

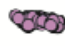

GDH2

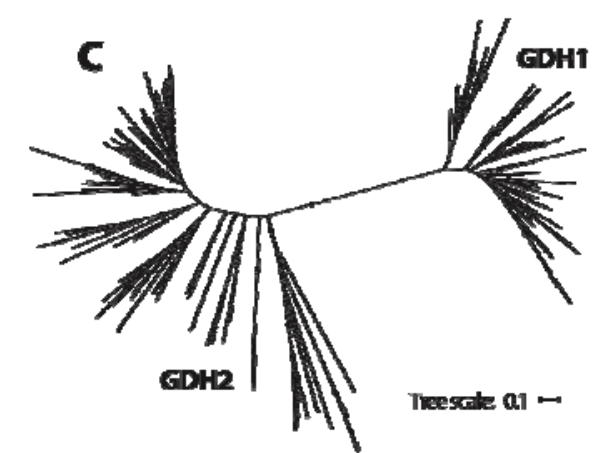

D ${ }_{1500}$

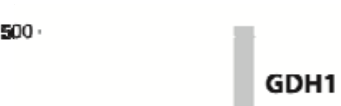

1000 .

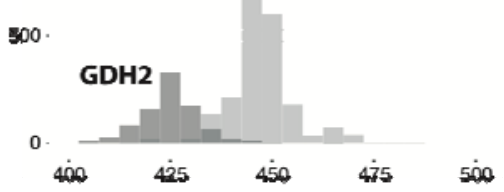

Figure 1. ASM-clust workflow overview and enolase superfamily example.

A) ASM-Clust workflow overview and B)

B) example of the ASM-Clust output on the structure

97 function linkage database (SFLD) enolase superfamily (48,850 sequences). The clusters are

98 colored by SFLD subgroup: enolase (red), galactarate dehydratase (orange), glucarate

99 dehydratase (light green), mandelate racemase (dark green), mannonate dehydratase (light blue),

100 methylaspartate-ammonia lyase (dark blue), muconate cycloisomerase (purple), muconate 
102 dehydratase' subgroup is split in two clusters, indicated with GDH1 and GDH2. C) Phylogenetic

103 analysis of the 'glucarate dehydratase' subgroup (clustered at 70\% identity), and D) sequence

104 length comparison confirms the clear separation of the two clusters.

105

\section{Results}

107 ASM-Clust was tested on the enolase superfamily in the gold-standard Structure Function 108 Linkage Database (SFLD) (Akiva et al. 2014). Sequences and annotation data table were 109 downloaded from the SFLD website (http://sfld.rbvi.ucsf.edu) and all 48,850 sequences were 110 clustered using ASM-Clust with default settings, and visualized using R (Fig 1b). The 111 'mannonate dehydratase' and 'muconate cycloisomerase (syn) like' subgroups, each containing 112 only a single isofunctional family, are well resolved. As expected, the functionally diverse 113 'muconate cycloisomerase' and 'mandelate racemase' subgroups each partition into multiple 114 discrete clusters (Figure 1b). The isofunctional 'enolase' and 'glucarate dehydratase' subgroups 115 also result in multiple clusters (Figure 1b). Phylogenetic analysis of the 'glucarate dehydratase' 116 subgroup confirms that the observed clusters respond to distinct clades that can also be separated 117 by sequence length (Figure 1c \& 1d, Supplementary methods). The smaller methylaspartate 118 ammonia-lyase and galactarate dehydratase subgroups (307 and 25 sequences respectively) are 119 more clearly resolved when ASM-clust is iteratively rerun on the "hub" cluster with a lower 120 perplexity value (Supplemental Figure S3). When prior high-quality annotation is not available, 121 clusters can be inspected for phylogeny, taxonomic distribution, and conserved residues to assess 122 whether they represent functionally divergent sequences.

123 ASM-Clust can retrieve clades from a complex superfamily with tens of thousands of sequences,

124 without prior reduction of the dataset. We expect this to become increasingly relevant as the 125 amount of sequence data from phylogenetically diverse organisms continues to grow rapidly, and 126 meaningful information can be overlooked while pre-clustering a sequence dataset.

\section{Funding}

129 This work was supported by the US Department of Energy, Office of Science, Office of 130 Biological and Environmental Research under award number DE-SC0016469 to Victoria J. 131 Orphan. Daan R. Speth was supported by the Netherlands Organisation for Scientific Research, 132 Rubicon award 019.153LW.039. 


\section{References}

Akiva, Eyal, Shoshana Brown, Daniel E. Almonacid, Alan E. Barber 2nd, Ashley F. Custer, Michael A. Hicks, Conrad C. Huang, et al. 2014. "The Structure-Function Linkage Database.”

137 Nucleic Acids Research 42 (Database issue): D521-30.

Altschul, S. F., W. Gish, W. Miller, E. W. Myers, and D. J. Lipman. 1990. "Basic Local Alignment Search Tool.” Journal of Molecular Biology 215 (3): 403-10.

Atkinson, Holly J., John H. Morris, Thomas E. Ferrin, and Patricia C. Babbitt. 2009. "Using

143 Sequence Similarity Networks for Visualization of Relationships across Diverse Protein 144 Superfamilies." PloS One 4 (2): e4345.

146 Borrel, Guillaume, Panagiotis S. Adam, Luke J. McKay, Lin-Xing Chen, Isabel Natalia Sierra-

147 García, Christian M. K. Sieber, Quentin Letourneur, et al. 2019. "Wide Diversity of Methane and

148 Short-Chain Alkane Metabolisms in Uncultured Archaea." Nature Microbiology 4 (4): 603-13.

150 Brown, Duncan P., Nandini Krishnamurthy, and Kimmen Sjölander. 2007. "Automated Protein

151 Subfamily Identification and Classification.” PLoS Computational Biology 3 (8): e160.

153 Buchfink, Benjamin, Chao Xie, and Daniel H. Huson. 2015. "Fast and Sensitive Protein 154 Alignment Using DIAMOND.” Nature Methods 12 (1): 59-60.

156 Copp, Janine N., Eyal Akiva, Patricia C. Babbitt, and Nobuhiko Tokuriki. 2018. "Revealing 157 Unexplored Sequence-Function Space Using Sequence Similarity Networks.” Biochemistry 57 158 (31): 4651-62.

160 Enright, A. J., S. Van Dongen, and C. A. Ouzounis. 2002. "An Efficient Algorithm for Large161 Scale Detection of Protein Families.” Nucleic Acids Research 30 (7): 1575-84. 
163 Ester, M., H. P. Kriegel, J. Sander, and X. Xu. 1996. "A Density-Based Algorithm for 164 Discovering Clusters in Large Spatial Databases with Noise.” KDD: Proceedings / International 165 Conference on Knowledge Discovery \& Data Mining. International Conference on Knowledge 166 Discovery \& Data Mining. https://www.aaai.org/Papers/KDD/1996/KDD96-037.pdf.

168 Finn, Robert D., Jody Clements, and Sean R. Eddy. 2011. "HMMER Web Server: Interactive 169 Sequence Similarity Searching.” Nucleic Acids Research 39 (Web Server issue): W29-37.

171 Hug, Laura A., Brett J. Baker, Karthik Anantharaman, Christopher T. Brown, Alexander J. 172 Probst, Cindy J. Castelle, Cristina N. Butterfield, et al. 2016. "A New View of the Tree of Life." 173 Nature Microbiology 1 (April): 16048.

175 Knutson, Stacy T., Brian M. Westwood, Janelle B. Leuthaeuser, Brandon E. Turner, Don 176 Nguyendac, Gabrielle Shea, Kiran Kumar, et al. 2017. "An Approach to Functionally Relevant 177 Clustering of the Protein Universe: Active Site Profile-Based Clustering of Protein Structures 178 and Sequences: Functionally Relevant Clustering of Protein Superfamilies.” Protein Science: A 179 Publication of the Protein Society 26 (4): 677-99.

181 Lee, David A., Robert Rentzsch, and Christine Orengo. 2010. "GeMMA: Functional Subfamily 182 Classification within Superfamilies of Predicted Protein Structural Domains." Nucleic Acids 183 Research 38 (3): 720-37.

185 Leuthaeuser, Janelle B., John H. Morris, Angela F. Harper, Thomas E. Ferrin, Patricia C. 186 Babbitt, and Jacquelyn S. Fetrow. 2016. "DASP3: Identification of Protein Sequences Belonging 187 to Functionally Relevant Groups.” BMC Bioinformatics 17 (1): 458.

189 Melo-Minardi, Raquel C. de, Karine Bastard, and François Artiguenave. 2010. "Identification of 190 Subfamily-Specific Sites Based on Active Sites Modeling and Clustering." Bioinformatics 26 191 (24): 3075-82. 
193 Schnoes, Alexandra M., Shoshana D. Brown, Igor Dodevski, and Patricia C. Babbitt. 2009.

194 "Annotation Error in Public Databases: Misannotation of Molecular Function in Enzyme 195 Superfamilies.” PLoS Computational Biology 5 (12): e1000605.

197 Steinegger, Martin, and Johannes Söding. 2017. "MMseqs2 Enables Sensitive Protein Sequence 198 Searching for the Analysis of Massive Data Sets." Nature Biotechnology 35 (11): 1026-28.

200 Van der Maaten, Laurens, and Geoffrey Hinton. 2008. "Visualizing Data Using T-SNE.” Journal 201 of Machine Learning Research: JMLR 9 (Nov): 2579-2605.

203 Van Der Maaten, L. 2014. “Accelerating T-SNE Using Tree-Based Algorithms.” Journal of 204 Machine Learning Research: JMLR.

205 http://www.jmlr.org/papers/volume15/vandermaaten14a/vandermaaten14a.pdf.

207 Zaremba-Niedzwiedzka, Katarzyna, Eva F. Caceres, Jimmy H. Saw, Disa Bäckström, Lina 208 Juzokaite, Emmelien Vancaester, Kiley W. Seitz, et al. 2017. "Asgard Archaea Illuminate the 209 Origin of Eukaryotic Cellular Complexity." Nature 541 (7637): 353-58. 
A 1) Subset $n$ sequences

2) Align $N$ sequences to subset $n$

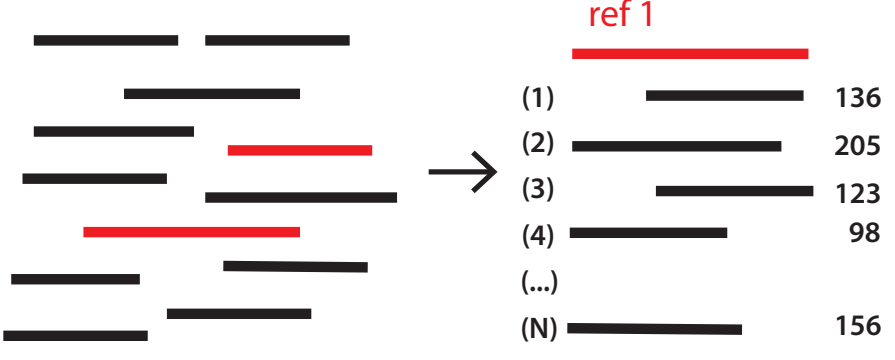

(...) ref $n$

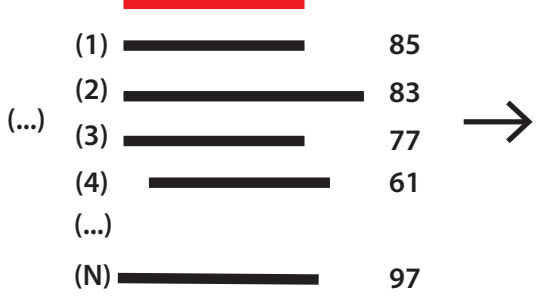

3) Build $N \times n$ Matrix

ref 1 ref 2 ref 3 (...) ref $n$

(1) 13600

(2) $205 \quad 124 \quad 0$

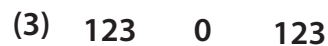

(4) $\begin{array}{lll}98 & 112 & 87\end{array}$

(...)

(N) $156 \quad 0 \quad 65$

97
B

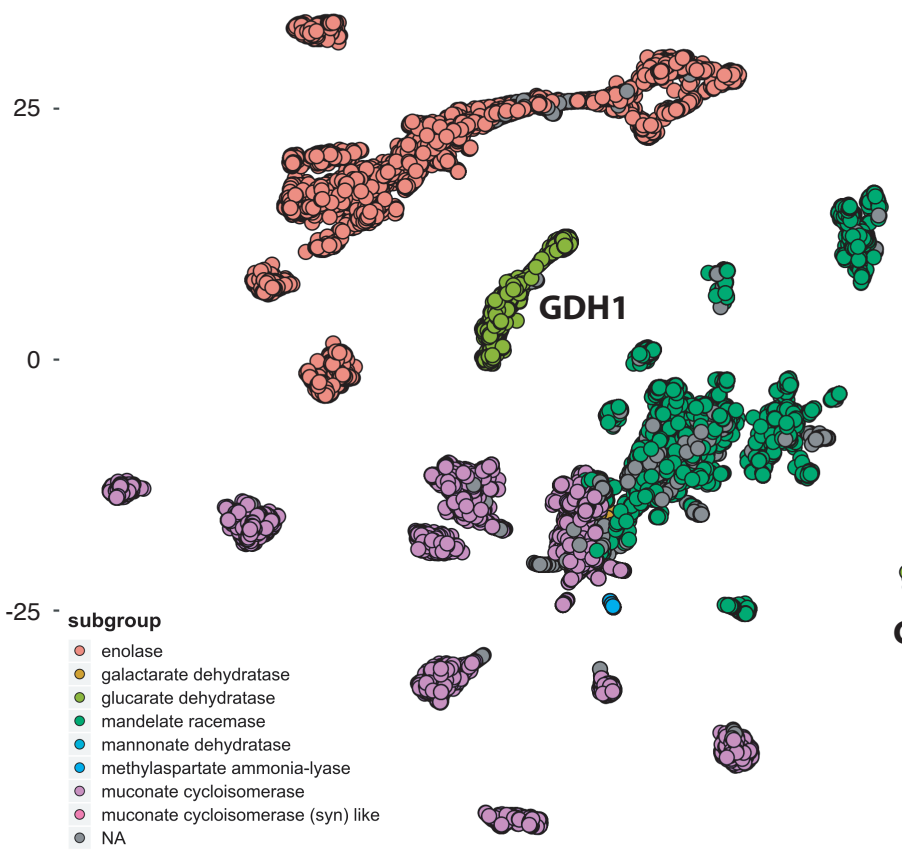

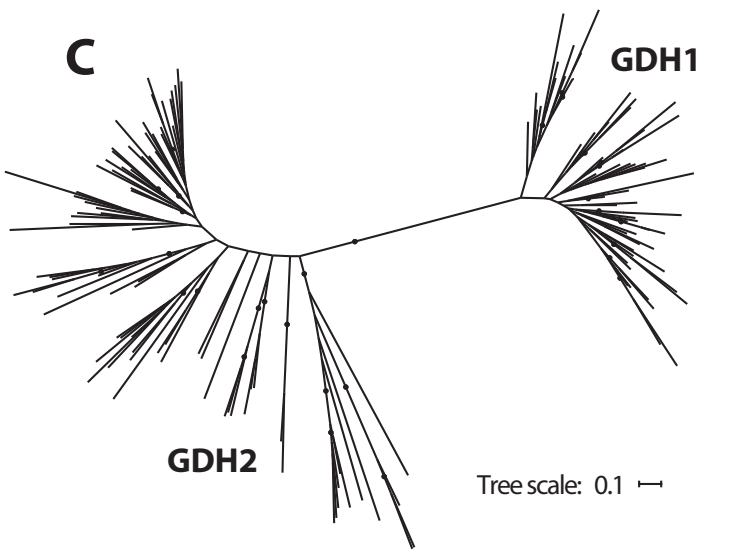

D

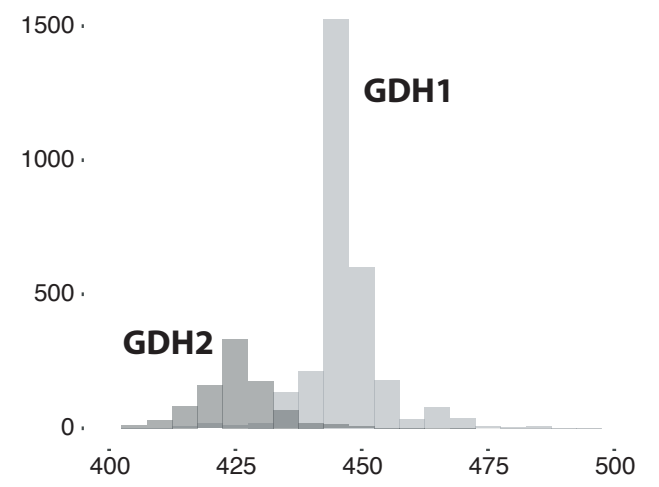

